TRANSACTIONS OF THE

AMERICAN MATHEMATICAL SOCIETY

Volume 284. Number 1. July 1984

\title{
MOMENTS OF BALANCED MEASURES ON JULIA SETS
}

BY

\author{
M. F. BARNSLEY ${ }^{1}$ AND A. N. HARRINGTON ${ }^{2}$
}

\begin{abstract}
By a theorem of S. Demko there exists a balanced measure on the Julia set of an arbitrary nonlinear rational transformation on the Riemann sphere. It is proved here that if the transformation admits an attractive or indifferent cycle, then there is a point with respect to which all the moments of a balanced measure exist; moreover, these moments can be calculated exactly. An explicit balanced measure is exhibited in an example where the Julia set is the whole sphere and for which the moments, with respect to any point, do not all exist.
\end{abstract}

1. Introduction. The Julia set for a nonlinear rational transformation on the Riemann sphere is the closure of the set of all repulsive cycles of the transformation $[\mathbf{B r}, \mathbf{F a}, \mathbf{J}]$. The "location" or "distribution" of the set is of interest in the theory of iterated maps, and it may be characterized by an associated invariant measure. In this paper, we extend previous results by showing that when there is an attractive or indifferent cycle, certain moments of such a measure exist and can be calculated exactly from the rational transformation. This means, for example, that when the Julia set is contained strictly in the real line, all of the orthogonal polynomials on the set can be calculated as can the entries of the corresponding infinite dimensional Jacobi matrices. The latter are of the type which have recently been shown to furnish model Schrödinger equations for crystals with almost periodic potentials [BBM].

This leads us to the following speculation, which provides one reason for our interest in the present topic. We note first the frequent occurrence, in the physical universe, of objects ("steady-state" systems), which are fractals from some point of view [Man]. We speculate consistently with Feigenbaum [Fe] and others (see [E]), that usually an important role in fixing the "shape" of such an object is played by some dominant iterative process. On the other hand, in line with the foundations of quantum mechanics, we have the expectation that in a probabalistic sense all of the observable characteristics of any object are determined by the spectra and eigenfunctions of appropriate linear operators, among which the Hamiltonian is the most important. For those objects for which the key to their structure is an iterative process, it should be possible to find model linear operators which on the one hand are built up from the iterative process and on the other hand predict the right kind of fractal structure for the object. Some of our work [BGH-1, BGH-2, BGH-3],

Received by the editors March 2, 1983 and, in revised form, October 18, 1983. Contents presented at 797th AMS Meeting, University of Maryland, College Park, October 30-31, 1982, by M. F. Barnsley.

1980 Mathematics Subject Classification. Primary 30D05, 30E05, 34A20.

Key words and phrases. Julia sets, orthogonal polynomials.

${ }^{1}$ Supported by NSF Grant MCS-8104862

${ }^{2}$ Supported by NSF Grant MCS- 8203325 
extended in the present paper, shows among other things how one may start with a rational map $T$ and find a selfadjoint operator whose spectrum is a fractal related to $T$, namely its Julia set.

If $T$ is a polynomial of degree greater than one then there exists a unique balanced measure $\mu$ on the Julia set $B$ [BGH-4, PK]. $\mu$ is the normalized electrostatic measure on $B$ and may be generated from the Böttcher equation at $\infty$ for $T$ [BGH-4]. In [BGH-1] we showed how one can recursively calculate the moments

$$
\int_{B} z^{n} d \mu(z), \quad n=0,1,2, \ldots
$$

Here we generalize to allow $T$ to be any rational function of degree greater than one, such that there exists an attractive or indifferent cycle. $z^{n}$ must be replaced by $(g(z))^{n}$ where $g(z)$ is any fractional linear transformation with its pole at a nonexpansive fixed point of $T$. The measure $\mu$ is supplied by a recent theorem of Demko [D], who has shown that balanced measures exist in a very general context. A special case of his result is that if $T$ is a rational function of degree $N$, then there exists a normalized measure $\mu$ supported on $B$, called a balanced measure for $T$, such that

$$
\mu T_{j}^{-1}(E)=\frac{1}{N} \mu(E) \text { for } j=1,2, \ldots, N
$$

where $E$ is any Borel subset of the Riemann sphere $\hat{\mathbf{C}}=\mathbf{C} \cup\{\infty\}$ and $\left\{T_{j}^{-1}\right.$ : $j=1,2, \ldots, N\}$ is a complete assignment of branches of the inverse of $T$. More detailed results in the specific context of iterated rational maps have been obtained by Friere, Lopes and Mañé [FLM], who show that $\mu$ is unique.

An interesting example of a balanced measure, which we will refer back to, is connected with Newton's method applied to $f(z)=\pi\left(1+z^{2}\right)$. The corresponding rational transformation is $T(z)=\left(z^{2}-1\right) / 2 z$, the Julia set is $B=\mathbf{R} \cup\{\infty\}$ and the balanced measure, which is absolutely continuous, is given by $d \mu(x)=d x / f(x)$. Thus, if Newton's method is applied on the real line to $f(x)$ then the probability density for visitation of points, starting from almost any point is $1 / f(x)$.

In $\S 2$, we consider the existence and evaluation of moments of the balanced measure. When both $B \subset \mathbf{R} \cup\{\infty\}$ and $\infty$ is an indifferent or attractive fixed point of $T$, the associated moment problem is shown to be determinate, and a selfadjoint operator, whose spectrum is $B$, is described.

In $\S 3$, we examine an example due to $M$. Lattès for which the Julia set is the whole of $\hat{\mathbf{C}}$. We are able to describe the balanced measure $\mu$ explicitly, and thus demonstrate that none of the moments $\int_{\hat{\mathbf{c}}}\left(g_{c}(z)\right)^{n} d \mu(z)$, for $n=2,3,4, \ldots$, exist, where $g_{c}(z)$ is any fractional linear transformation with its pole at any point $c \in \hat{\mathbf{C}}$.

2. Existence and evaluation of moments. We define the expansion factor at a fixed point $c$ of $T$ by

$$
E(T, c)= \begin{cases}\left|T^{\prime}(c)\right| & \text { if } c \neq \infty, \\ \lim _{z \rightarrow \infty}\left|\frac{z}{T(z)}\right| & \text { if } c=\infty .\end{cases}
$$


Notice that

$$
E\left(\psi T \psi^{-1}, \psi c\right)=E(T, c)
$$

when $\psi$ is a fractional linear transformation.

TheOREM 1. Suppose $T: \hat{\mathbf{C}} \rightarrow \hat{\mathbf{C}}$ is a rational transformation of degree $N>1, c$ is a fixed point of $T, g$ is a fractional linear transformation with pole $c$ and $\mu$ is the balanced measure for $T$. Then the moment integral

$$
M_{n}=\int_{\hat{\mathbf{C}}}(g(z))^{n} d \mu(z)
$$

exists for positive integer $n$ if and only if

$$
E(T, c)<\sqrt[n]{N}
$$

Proof. Without loss of generality, we take $c=0$, so that $T(0)=0$ and we can choose $g(z)=1 / z$. For $R>0$, let $C(R)$ denote the disk $\{z:|z| \leqslant R\}$.

Suppose $0 \leqslant E(T, 0)<N^{1 / n}$. If $E(T, 0)<1$, then there is a disk $C(D)$ of radius $D>0$ which does not intersect the Julia set. Hence

$$
\int_{\hat{\mathbf{C}}} \frac{d \mu}{\left|z^{m}\right|} \leqslant \frac{1}{D^{m}} \int_{\hat{\mathbf{C}}} d \mu=\frac{1}{D^{m}}<\infty .
$$

On the other hand, if $1 \leqslant E(T, 0)<N^{1 / n}$ then we can find constants $K$ and $R>0$ so that

$$
N^{-1 / n}<\frac{1}{K}<\left|T_{1}^{-1^{\prime}}(z)\right| \leqslant 1 \text { for all } z \in C(R),
$$

and

$$
C\left(K^{-j} R\right) \subset T_{1}^{-j} C(R), \quad j=1,2,3, \ldots,
$$

where $T_{1}^{-1}(z)$ is the branch of $T^{-1}$ such that $T_{1}^{-1}(0)=0$, and $T_{1}^{-(k+1)}(z)=$ $T_{1}^{-1}\left(T_{1}^{-k}(z)\right)$ for $k=1,2,3, \ldots$ (Note that $T_{1}^{-1}(z)$ is analytic in a neighborhood of 0.) It follows that

$$
\mu\left(C\left(K^{-j} R\right)\right) \leqslant \mu\left(T_{1}^{-j} C(R)\right)=N^{-j} \mu(C(R)),
$$

where in the last step we have used the balanced property of $\mu$. We now have

$$
\begin{aligned}
\int_{C(R)} \frac{d \mu}{\left|z^{n}\right|} & =\sum_{j=0}^{\infty} \int_{C\left(K^{-j} R\right) \backslash C\left(K^{-j-1} R\right)} \frac{d \mu}{\left|z^{n}\right|} \\
& \leqslant \sum_{j=0}^{\infty} \frac{1}{\left(K^{-j-1} R\right)^{n}} \mu\left(C\left(K^{-j} R\right)\right) \\
& \leqslant \mu(C(R))(K / R)^{n} \sum_{j=0}^{\infty}\left(K^{n} / N\right)^{j}<\infty .
\end{aligned}
$$

The convergence of $\int_{\hat{\mathbf{C}}} d \mu /\left|z^{n}\right|$ follows at once.

To prove the converse, we suppose $N^{1 / n} \leqslant\left|T^{\prime}(0)\right|<\infty$. Fix a neighborhood $\tilde{E}$ of 0 and $\alpha>0$ so that

$$
(\neq) \quad\left|T_{1}^{-1^{\prime}}(z)\right| \leqslant N^{-1 / n}+\alpha|z| \text { for all } z \in \tilde{E} \text {. }
$$


Let $E$ be a neighborhood of 0 which is contained in $\tilde{E}$, and let

$$
R_{j}=\sup \left\{\left|T_{1}^{-j} z\right|: z \in E\right\} .
$$

We can suppose $R_{0}$ is so small that $1-A-B R_{0}>0$, where $A=N^{-1 / n}$ and $B=\alpha / 2 A$. Then with the aid of $(\neq)$ and integration from 0 to $T_{1}^{-j}(z)$ we can establish that

$$
R_{j+1} \leqslant A R_{j} /\left(1-B R_{j}\right) \text { for } j=0,1,2, \ldots
$$

By induction we can now prove

$$
R_{j} \leqslant K_{2} A^{j} /\left(1+K_{1} A^{j}\right) \text { for } j=0,1,2, \ldots,
$$

where

$$
K_{1}=B R_{0} /\left(1-A-B R_{0}\right) \text { and } K_{2}=(1-A) K_{1} / B \text {. }
$$

Let $U_{0}=E \backslash T_{1}^{-1} E$ and $U_{j}=T_{1}^{-j} U_{0}$ for $j=1,2, \ldots$ Then

$$
\begin{aligned}
\int_{E} \frac{d \mu}{\left|z^{n}\right|} & =\sum_{l=0}^{\infty} \int_{U_{l}} \frac{d \mu}{|z|^{n}} \geqslant \sum_{l=0}^{\infty} \frac{1}{R_{l}^{n}} \mu\left(U_{l}\right) \\
& =\sum_{l=0}^{\infty} \frac{\mu\left(U_{0}\right)}{N^{l} R_{l}^{n}} \geqslant \frac{\mu\left(U_{0}\right)}{K_{2}^{n}} \sum_{l=0}^{\infty} \frac{1}{N^{l} A^{l n}},
\end{aligned}
$$

where we have again used the balanced property of $\mu$. The latter sum diverges, whence so does $M_{n}$.

THEOREM 2. When the moment integral $M_{n}$ in Theorem 1 exists it can be exactly calculated as follows. Let

$$
\begin{gathered}
g T g^{-1}=\frac{z^{N}+a_{1} z^{N-1}+a_{2} z^{N-2}+\cdots+a_{N}}{b_{1} z^{N-1}+b_{2} z^{N-2}+\cdots+b_{N}}, \\
C_{k}(z)= \begin{cases}0 & \text { for } k=N+1, N+2, \ldots, \\
a_{k}-z b_{k} & \text { for } k=1,2, \ldots, N,\end{cases} \\
S_{k}(z)=-k C_{k}(z)-\sum_{j=1}^{k-1} S_{k-j}(z) C_{j}(z) \quad \text { for } k=1,2, \ldots, n .
\end{gathered}
$$

Write

$$
S_{m}(z)=\sum_{j=0}^{m} r_{m j} z^{j}
$$

Then

$$
M_{m}=\frac{1}{N-b_{1}^{m}} \sum_{j=0}^{m-1} r_{m j} M_{j} \text { for } m=1,2, \ldots, n
$$

where $M_{0}=1$. 
Proof. Let $R=g T g^{-1}$. Then $R$ is expressible in the form (1) because $\infty$ must be a fixed point of $R$. The expansion factor of $R$ at $\infty$ is

$$
\lim _{z \rightarrow \infty}\left|\frac{z}{R(z)}\right|=\left|b_{1}\right|=E\left(g T g^{-1}, g c\right)=E(T, c)<\sqrt[n]{N} .
$$

Since $M_{n}$ exists, so also does $M_{m}$ for $m=1,2, \ldots, n-1$. The balanced measure $\sigma$ for $R$ is specified by $d \sigma(z)=d \mu\left(g^{-1}(z)\right)$, and we have

$$
M_{m}=\int_{\hat{\mathbf{C}}}(g(z))^{m} d \mu(z)=\int_{\hat{\mathbf{C}}} z^{m} d \sigma(z) .
$$

Using the balanced property of $\sigma(z)$ we have

$$
M_{m}=\int_{\hat{\mathbf{C}}} z^{m} d \sigma(z)=\frac{1}{N} \int_{\hat{\mathbf{C}}_{j=1}} \sum_{j=1}^{N}\left(R_{j}^{-1}(z)\right)^{m} d \sigma(z)
$$

where $\left\{R_{j}^{-1}(z)\right\}_{j=1}^{N}$ is a complete assignment of branches of the inverse of $R$. But

$$
S_{m}(z)=\sum_{j=1}^{N}\left(R_{j}^{-1}(z)\right)^{m}
$$

is just the $m$ th symmetric function associated with the roots of the polynomial in $W$,

$$
W^{N}+c_{1}(z) W^{N-1}+\cdots+c_{N}(z) \text {. }
$$

The symmetric functions $\left\{S_{m}(z)\right\}_{m=0}^{n}$ can be calculated successively using (2), [G]. It is quickly verified that $S_{m}(z)$ is a polynomial in $z$ of degree at most $m$, and can be written as in (3) where the $r_{m j}$ 's are independent of $z$.

We can now rewrite (6) as

$$
N M_{m}=\int_{\hat{\mathbf{C}}} S_{m}(z) d \sigma(z)=\sum_{j=0}^{m} r_{m j} M_{j} .
$$

Observing that $r_{m m}=b_{1}^{m}$, and recalling from (5) that $\left|b_{1}\right|^{m}<N$ for $m=1,2, \ldots, n$, we now obtain (4) as desired.

EXAMPLE. Let $T(z)=\left(z^{2}-1\right) / z$. Then $\infty$ is an indifferent fixed point and we can choose $g(z)=z$. Since any real point has only real inverse images under $T$, it follows that $B \subset \mathbf{R} \cup\{\infty\}$. By Theorem 1 all of the moments $M_{n}=\int_{\mathbf{R}} x^{n} d \mu(x)$ exist. Following Theorem 2, we find $c_{1}(x)=-z$ and $c_{2}(z)=-1$, so $S_{1}(z)=z$, $S_{2}(z)=z^{2}+2$, and $S_{n}(z)=z S_{n-1}(z)+S_{n-2}(z)$ for $n=3,4,5, \ldots$. Hence $M_{2 k+1}$ $=0$ for $k=0,1,2, \ldots$ (which says that $\mu$ is symmetric about the origin) and $M_{0}=1$, $M_{2}=2, M_{4}=10, M_{6}=80, M_{8}=874, M_{10}=12,092, M_{12}=202,384$, and $M_{14}=$ $3,973,580$ and so on. Using the moments we readily calculate the successive orthogonal polynomials $\left\{P_{n}(x)\right\}$ which obey

$$
\int_{\mathbf{R}} P_{n}(x) P_{m}(x) d \mu(x)=0 \text { for } n \neq m,
$$

where $P_{n}(x)$ is of degree $n$ and has leading coefficient 1 . We obtain $P_{0}(z)=1$, $P_{1}(z)=z, P_{2}(z)=z^{2}-2, P_{3}(z)=z^{3}-5 z, P_{4}(z)=z^{4}-10 z^{2}+10, P_{5}(z)=z^{5}$ $-158 z^{3} / 10+39 z$ and so on. These polynomials obey the three-term recurrence 
formula $P_{n+1}=z P_{n}(z)-a_{n}^{2} P_{n-1}(z)$ for $n=1,2,3, \ldots$, where $a_{1}^{2}=2, a_{2}^{2}=3, a_{3}^{2}=$ $5, a_{4}^{2}=29 / 5, \ldots$. Thus, the associated Jacobi matrix is

$$
J=\left(\begin{array}{cccccc}
0 & \sqrt{2} & 0 & 0 & 0 & . \\
\sqrt{2} & 0 & \sqrt{3} & 0 & 0 & . \\
0 & \sqrt{3} & 0 & \sqrt{5} & 0 & . \\
0 & 0 & \sqrt{5} & 0 & \sqrt{29 / 5} & . \\
0 & 0 & 0 & \sqrt{29 / 5} & 0 & . \\
\cdot & . & . & \cdot & . & .
\end{array}\right)
$$

It is a consequence of the next theorem that $J$ corresponds to a selfadjoint operator in $l_{2}$, whose spectrum is $B$ and whose spectral density is $\mu$. Observe that if we multiply the transformation $T(z)$ by $1 / 2$, then $\infty$ becomes a repulsive fixed point and the balanced measure becomes the one mentioned in the introduction. None of the moments, $M_{n}, n=1,2,3, \ldots$, then exist.

In situations like the above example, where one can associate a symmetric Jacobi matrix $J$ with the orthogonal polynomials, one would like to know the relationship between $J$ and the original iterated map. This relationship is immediate if one can establish that the moments $M_{n}=0,1,2, \ldots$ uniquely determine the balanced measure $\mu$.

THeOREM 3. If $T: \hat{\mathbf{C}} \rightarrow \hat{\mathbf{C}}$ is a rational transformation with Julia set $B \subset \mathbf{R} \cup\{\infty\}$, $\infty$ is a fixed point of $T, E(T, \infty) \leqslant 1$, and $\mu$ is the balanced measure for $T$, then the moment problem

$$
M_{n}=\int_{-\infty}^{+\infty} x^{n} d \mu(x) \text { for } n=0,1,2, \ldots
$$

is determinate.

COROLlaRY. If $J$ is the symmetric Jacobi matrix associated with the moment problem in Theorem 3, then $J$ possesses a unique selfadjoint extension $\bar{J}$ with domain in $l_{2}$, the Hilbert space of square-summable sequences. The spectrum of $\bar{J}$ is $B$, and its spectral density is $\mu$.

Proof of Theorem 3. If $E(T, \infty)<1$, then $\infty$ is an attractive fixed point for $T(z)$ and we can find $R \in(0, \infty)$ such that $B \subset\{z:|z|<R\}$. In this case

$$
\left|M_{n}\right|=\left|\int_{-\infty}^{+\infty} x^{n} d \mu(x)\right| \leqslant R^{n} \text { for } n=0,1,2, \ldots
$$

If $E(T, \infty)=1$, then there are constants $R_{0}>0$ and $C>0$ such that

$$
\left|T_{1}^{-1}(z)\right| \leqslant\left(1+\frac{C}{|z|}\right) \cdot|z| \text { for }|z| \geqslant R_{0} .
$$

Let $R_{n}=n C / \varepsilon$ where $\varepsilon$ is chosen small and positive, so that $R_{n}>R_{0}$ and $\exp (\varepsilon)<N$, where $N$ is the degree of $T(z)$. Then

$$
\left|T_{1}^{-1}(z)\right| \leqslant k_{n}|z| \text { for }|z| \geqslant R_{n},
$$


where $k_{n}=(1+\varepsilon / n)$. If we introduced the notation $A(R)=\{z \in \hat{\mathbf{C}}:|z| \geqslant R\}$, then it follows that

$$
T_{1}^{-j} A\left(R_{n}\right) \supset A\left(k_{n}^{j} R_{n}\right) \text { for } j=0,1,2, \ldots
$$

Hence, proceeding much as we did for the proof of Theorem 1, we find

$$
\begin{aligned}
\left|M_{n}\right| & =\left|\int_{\hat{\mathbf{C}}} z^{n} d \mu(z)\right| \leqslant \int_{|z| \leqslant R_{n}}|z|^{n} d \mu+\sum_{j=0}^{\infty} \int_{A\left(k_{n}^{j+1} R_{n}\right) \backslash A\left(k_{n}^{j} R_{n}\right)}|z|^{n} d \mu \\
& \leqslant R_{n}^{n}+\sum_{j=0}^{\infty}\left(k_{n}^{j+1} R_{n}\right)^{n} \mu\left(A\left(k_{n}^{j} R_{n}\right)\right) \\
& \leqslant R_{n}^{n}+R_{n}^{n} k_{n}^{n} \sum_{j=0}^{\infty}\left(k_{n}^{n}\right)^{j} N^{-j} .
\end{aligned}
$$

Then noting that $k_{n}^{n}=(1+\varepsilon n)^{n}<\exp (\varepsilon)<N$, we have

$$
\left|M_{n}\right| \leqslant \frac{C^{n} n^{n}}{\varepsilon^{n}} \cdot\left(1+N^{2} /(N-\exp (\varepsilon))\right)
$$

for $n=0,1,2, \ldots$, where $C$ and $\varepsilon$ do not depend upon $n$.

From (i) and (ii) above, it follows that there exists a finite constant, independent of $n$, such that

$$
\sqrt[n]{\left|M_{n}\right|} \leqslant n \cdot \text { constant } \text { for } n=1,2, \ldots
$$

Hence $\sum_{n=0}^{\infty} 1 / \sqrt[2 n]{M_{2 n}}$ is divergent, which is just Carleman's condition for the moment problem to be determinate [W].

Sketch of Proof of Corollary. Let $f_{0} \in l_{2}$ be represented by the column vector $f_{0}=(1,0,0, \ldots)^{T}$, where $T$ denotes the transpose, and let $f_{n}=J^{n} f_{0}$ for $n=1,2,3, \ldots$ Express the inner product between $g$ and $h$ in $l_{2}$ by $\langle g, h\rangle$, and write $\langle g, g\rangle=\|g\|^{2}$. Then, with some algebra, see for example [Ak, Chapter 4], one can show

$$
M_{n}=\left\langle f_{0}, f_{n}\right\rangle \text { for } n=0,1,2, \ldots
$$

From (*) it follows that the series

$$
\sum_{m=0}^{\infty} \frac{\left\|J^{n} f_{n}\right\|}{m !} z^{m}=\sum_{m=0}^{\infty} \frac{\sqrt{M_{2 m+2 n}}}{m !} z^{m}
$$

has nonzero radius of convegence, which says that $f_{n}$ is an analytic vector for $J$. Let $L$ denote the linear manifold which consists of all finite linear combinations of $\left\{f_{n}\right.$ : $n=0,1,2, \ldots\}$. Then every element of $L$ is an analytic vector for $J$. Also $L$ is dense in $l_{2}$, and hence, by Nelson's theorem (see for example [Mas]), the closure of $J$ is selfadjoint. The assertion that $\mu$ is the spectral density for $\bar{J}$ follows from classical moment theory, see [Ak, Theorem 4.1.3].

As long as the complement of the Julia set contains a Fatou domain or a Siegel disk, it is always possible to find an attractive or indifferent $k$-cycle for $T(z)$, see [Su]. That is, there exists a strictly positive integer $k$ and a point $c \in \hat{\mathbf{C}}$ such that 
$T^{k}(c)=c$ and $E\left(T^{k}, c\right) \leqslant 1$, where $T^{1}(z)=T(z), T^{2}(z)=T(T(z)), T^{3}(z)=$ $T(T(T(z))), \ldots$. Since both $T(z)$ and $T^{k}(z)$ have the same Julia set and the same balanced measure, Theorem 1 remains true with " $c$ is a fixed point of $T$ " replaced by " $c$ is a fixed point of $T^{k}$ " and $E(T, c)$ replaced by $E\left(T^{k}, c\right)$. In this case, when the moments exist, they can be calculated just as in Theorem 2, but with $g T g^{-1}$ replaced by $g T^{k} g^{-1}$.

For example, if $c$ is a fixed point of $T^{k}$ 'with $E\left(T^{k}, c\right) \leqslant 1$, and if $\psi(z)$ is any rational function whose poles are located at points belonging to the set $\left\{c, T(c), T^{2}(c), \ldots, T^{k-1}(c)\right\}$, then $\int_{C} \psi(z) d \mu(z)$ exists, and can be calculated explicitly in terms of the coefficients in $\psi$ and $T$.

Another example concerns the map $T(z)=z^{2}-1$ which admits the superstable two-cycle $\{0,1\} . T^{2}(z)$ possesses the fixed point $c=0$, which is mapped to $\infty$ by $g(z)=1 / z$. Then we find $g T^{2} g^{-1}=z^{4} /\left(1-2 z^{2}\right)$, corresponding to which $S_{1}=0$, $S_{2}=-4 z, \quad S_{3}=0, \quad S_{4}=4 z \cdot(2 z+1), \quad S_{k+5}=-2 z \cdot S_{k+3}+z \cdot S_{k+1} \quad(k=$ $0,1,2, \ldots)$, and consequently, $M_{n}=\int_{\mathbf{C}} z^{-n} d \mu(z)=0$ for $n=1,2,3, \ldots$ The same conclusion also follows from an integration formula given in [BGH-5].

3. The example of M. Lattès. We have shown that if there is an indifferent or attractive cycle, then one can find $c \in \hat{\mathbf{C}}$ such that the moments $M_{n}=$ $\int_{\hat{\mathbf{c}}}\left(g_{c}(z)\right)^{n} d \mu(z)$ exist for all $n=0,1,2, \ldots$, where $g_{c}(z)$ denotes any fractional linear transformation with pole at $c$. Here we consider the example of the transformation $T(z)=L(z)$, where

$$
L(z)=\frac{\left(z^{2}+1\right)^{2}}{4 z\left(z^{2}-1\right)},
$$

for which $\mathbf{M}$. Lattès $[\mathbf{L}]$ has noted $B=\hat{\mathbf{C}}$. We show that the balanced measure for $L(z)$ is

$$
d \mu(z)=\alpha \cdot \frac{d x d y}{\sqrt{\left|z \cdot\left(z^{2}-1\right)\right|}},
$$

where $\alpha \in(0, \infty)$ is fixed by the condition $M_{0}=1$. Hence, in this case, none of the moments $M_{n}$ for $n=2,3,4, \ldots$, exist, for any $c \in \hat{\mathbf{C}}$.

We begin by recalling why $B=\hat{\mathbf{C}}$ for $L(z)$. Let $P(z)$ denote the Weierstrass function which obeys the first order ordinary differential equation

$$
P^{\prime}(z)^{2}=4 P(z)^{3}-4 P(z) .
$$

Note than any Weierstrass function obeys the identity

$$
P(2 z)=\frac{1}{4}\left(\frac{P^{\prime \prime}(z)}{P^{\prime}(z)}\right)^{2}-2 P(z),
$$

see [Ah]. On substituting from (8) into (9), we find

$$
P(2 z)=L(P(z)) .
$$

(This is essentially the Poincare equation for $L$, associated with the fixed point $\infty$, where the expansion factor is 4 : the apparent mystery that (10) involves 2 rather 
than 4 is resolved when on realizes that the Poincare function in this case is $\theta(z)=P(\sqrt{z})$, and $(10)$ can be rewritten $\theta(4 z)=L(\theta(z))$. Notice that $\theta(z)$ is a meromorphic function because $P(z)$ is an even meromorphic function.) From (10) it follows that $P\left(2^{n} z\right)=L^{n}(P(z))$ for $n=1,2,3, \ldots$, and hence it is possible to give a complete description of what happens when $L$ is applied iteratively.

$P$ is a doubly periodic function with periods $\omega$ and $i \omega$, and hence can be regarded as a function defined on the torus. The map $z \rightarrow 2 z$ on the torus, being expanding, clearly has no proper invariant subsets, so neither has $L$. It follows that the Julia set of $L$ must be $\hat{\mathbf{C}}$.

Let the torus be made from the square $S$ with vertices $0, \omega, \omega+i \omega, i \omega$. Then the normalized invariant measure for $z \rightarrow 2 z$ on $S$ is the uniform one, namely $d A / \omega^{2}$ where $d A$ denotes a differential area element at $z$ in $S$. Since $P$ maps $S$ two-to-one onto $\hat{\mathbf{C}}$, the corresponding invariant measure for $L$ is given by $d \mu(P(z))=2$. $d A /\left|P^{\prime}(z)\right| \cdot \omega^{2}$ where $d A$ denotes a differential area element at $P(z)$ in $\hat{\mathbf{C}}$. Letting $w=P(z)$, and using (7), this measure can be re-expressed

$$
d \mu(w)=d A / \omega^{2}\left|w \cdot\left(w^{2}-1\right)\right|, \quad \text { at } w \in \hat{\mathbf{C}} .
$$

By construction, the measure is normalized, and so we must have $\alpha=1 / \omega^{2}$ in (7). This gives one way to find $\omega$. Alternatively, one can consult [By], wherefrom we find $\omega=2.240 \ldots$ One can check straightforwardly that $\mu$ is balanced; hence $\mu$ is the balanced measure for $L$.

Thus, we have shown that $L(z)$ is an example for which the Julia set is $\hat{\mathbf{C}}$, and for which none of the moments $\int_{\hat{\mathbf{c}}}\left(g_{c}(z)\right)^{n} d \mu(z), n=2,3,4, \ldots$, exist, regardless of the choice of $c \in \hat{\mathbf{C}}$.

There exist other examples, due to Herman $[\mathbf{H}]$, for which the Julia set in $\hat{\mathbf{C}}$, and for which infinity is an indifferent fixed point. For these all of the moments $\int_{\hat{\mathbf{C}}} z^{n} d \mu(z), n=0,1,2, \ldots$, must exist and can be calculated explicitly.

ACKNOWLEDGEMENTS. We thank the referee for this help in revising Section 3 and other useful comments.

\section{REFERENCES}

[Ah] L. V. Ahlfors, Complex analysis, McGraw-Hill, New York, 1979.

[Ak] N. I. Akhiezer, The classical moment problem, Hafner, New York, 1965.

[BGH-1] M. F. Barnsley, J. S. Geronimo and A. N. Harrington, Orthogonal polynomials associated with invariant measures on Julia sets, Bull. Amer. Math. Soc. 7 (1982), 381-384.

[BGH-2] _ , On the invariant sets of a family of quadratic maps, Comm. Math. Phys. 88 (1983), 479-501.

[BGH-3] _ Infinite dimensional Jacobi matrices associated with Julia sets, Proc. Amer. Math. Soc. 88 (1983), 625-630.

[BGH-4] _ Geometry electrostatic measure and orthogonal polynomials on Julia sets for polynomials, Ergodic Theory Dynamical Systems (to appear).

[BGH-5] __ Geometrical and electrical properties of some Julia sets, Stat. Phys. (submitted).

[BBM] J. Bellissard, D. Bessis and P. Moussa, Chaotic states for almost periodic Schrödinger operators, Phys. Rev. Lett. 49 (1982), 701-704.

[Br] H. Brolin, Invariant sets under iteration of rational functions, Ark. Mat. 6 (1965), 103-144.

[By] P. F. Byrd and M. D. Friedman, Handbook of elliptic integrals for engineers and physicists, Springer-Verlag, New York, 1954.

[D] S. G. Demko, On balanced measures, preprint. 
[E] J. P. Eckmann, Roads to turbulence in dissipative dynamical systems, Rev. Modern Phys. 53 (1981), 643-654.

[Fa] M. P. Fatou, Sur les equations fonctionelles, Bull. Soc. Math. France 47 (1919), 161-271; 48, 33-94, 208-314.

[Fe] M. Feigenbaum, Quantitative universality for a class of nonlinear transformations, J. Statist. Phys. 19 (1978), 25-52.

[FLM] A. Freire, A. Lopes and R. Mañe, An invariant measure for rational maps, 1982, preprint.

[G] L. Gaal, Classical Galois theory with examples, Markham, Chicago, Ill., 1971.

[H] M. R. Herman, Exemples de fractions rationelles ayant une orbite dense sur la sphere de Riemann, Ecole Polytechnique, 1983, preprint.

[J] G. Julia, Memoire sur l'iteration des fonctions rationelles, J. Math. Pures Appl. 4 (1918), 47-245.

[L] S. Lattès, Sur l'iteration des substitutions rationelles et les fonctions de Poincaré, C. R. Acad. Sci. Paris 166 (1918), 26-28.

[Man] B. Mandelbrot, Fractals: Form, chance and dimension, Freeman, San Francisco, Calif., 1977.

[Mas] D. Masson, Hilbert space and the Padé approximant, in The Pade Approximant in Theoretical Physics (Edited by G. A. Baker, Jr. and J. L. Gammel), Academic Press, New York and London, 1970, pp. 197-218.

[PK] T. S. Pitcher and J. R. Kinney, Some connections between ergodic theory and iteration of polynomials, Ark. Mat. 8 (1968), 25-32.

[Su] D. Sullivan, Iteration des fonctions analytiques complexes, C. R. Acad. Sci. Paris 294 (1982), 301.

[W] H. S. Wall, Analytic theory of continued fractions, Van Nostrand, Princeton, N. J., 1958.

School of Mathematics, Georgia Institute of Technology, Atlanta, Georgia 30332 (Current address of M. F. Barnsley)

Current address (A. N. Harrington): Department of Mathematical Sciences, Loyola University, Chicago, Illinois 60626. 